JOURNAL OF THE

AMERICAN MATHEMATICAL SOCIETY

Volume 10, Number 2, April 1997, Pages 327-349

S 0894-0347(97)00225-7

\title{
GENERALIZATIONS OF MÜNTZ'S THEOREM VIA A REMEZ-TYPE INEQUALITY FOR MÜNTZ SPACES
}

\author{
PETER BORWEIN AND TAMÁS ERDÉLYI \\ Dedicated to the memory of Paul Erdös
}

\section{INTRODUCTION}

Müntz's beautiful, classical theorem characterizes sequences $\Lambda:=\left(\lambda_{i}\right)_{i=0}^{\infty}$ with

$$
0=\lambda_{0}<\lambda_{1}<\lambda_{2}<\cdots
$$

for which the Müntz space $M(\Lambda):=\operatorname{span}\left\{x^{\lambda_{0}}, x^{\lambda_{1}}, \ldots\right\}$ is dense in $C[0,1]$. Here, and in what follows, $\operatorname{span}\left\{x^{\lambda_{0}}, x^{\lambda_{1}}, \ldots\right\}$ denotes the collection of finite linear combinations of the functions $x^{\lambda_{0}}, x^{\lambda_{1}}, \ldots$ with real coefficients, and $C(A)$ is the space of all real-valued continuous functions on $A \subset[0, \infty)$ equipped with the uniform norm. If $A:=[a, b]$ is a finite closed interval, then the notation $C[a, b]:=C([a, b])$ will be used. Throughout this paper $\Lambda:=\left(\lambda_{i}\right)_{i=0}^{\infty}$ denotes a sequence satisfying (1.1). Müntz's Theorem ([11], [18], [27], [30]) states the following.

Theorem. $M(\Lambda)$ is dense in $C[0,1]$ if and only if $\sum_{i=1}^{\infty} 1 / \lambda_{i}=\infty$.

The original Müntz Theorem proved by Müntz [18] in 1914, by Szász [27] in 1916, and anticipated by Bernstein [3] was only for sequences of exponents tending to infinity. The point 0 is special in the study of Müntz spaces. Even replacing $[0,1]$ by an interval $[a, b] \subset[0, \infty)$ in Müntz's Theorem is a non-trivial issue. This is, in large measure, due to Clarkson and Erdős [12] and Schwartz [24] whose works include the result that if $\sum_{i=1}^{\infty} 1 / \lambda_{i}<\infty$, then every function belonging to the uniform closure of $M(\Lambda)$ on $[a, b]$ can be extended analytically throughout the region

$$
\{z \in \mathbb{C} \backslash(-\infty, 0]:|z|<b\} .
$$

There are many generalizations and variations of Müntz's Theorem ([1], [4], [5], [6], [7], [8], [9], [17], [19], [24], [26], [28], [29]). There are also still many open problems. The proper generalizations to many variables are still open.

In Section 6 of this paper we show that the interval $[0,1]$ in Müntz's Theorem can be replaced by an arbitrary compact set $A \subset[0, \infty)$ of positive Lebesgue measure.

Received by the editors June 10, 1994 and, in revised form, September 20, 1996.

1991 Mathematics Subject Classification. Primary 41A17; Secondary 30B10, 26 D15.

Key words and phrases. Remez inequality, Müntz's Theorem, Müntz spaces, Dirichlet sums, density.

Research of the first author was supported, in part, by NSERC of Canada. Research of the second author was supported, in part, by NSF under Grant No. DMS-9024901 and conducted while an NSERC International Fellow at Simon Fraser University. 
That is, if $A \subset[0, \infty)$ is a compact set of positive Lebesgue measure, then $M(\Lambda)$ is dense in $C(A)$ if and only if $\sum_{i=1}^{\infty} 1 / \lambda_{i}=\infty$.

If $A$ contains an interval, then this follows from the already mentioned results of Clarkson, Erdős, and Schwartz. However, their results and methods cannot handle the case that, for example, $A \subset[0,1]$ is a Cantor-type set of positive measure. Note also that the scaling $x \rightarrow \alpha x$ reduces the case that $A \subset[0, \infty)$ is compact to the case that $A \subset[0,1]$ is closed, so working on $[0,1]$ is perfectly general.

In the case that $\sum_{i=1}^{\infty} 1 / \lambda_{i}<\infty$, analyticity properties of the functions belonging to the uniform closure of $M(\Lambda)$ on $A$ are also established.

An analogue of the above result is also proved in $L_{w}^{q}(A)$, where $w$ is a nonnegative integrable weight function on $A$ with $\int_{A} w>0$, and $q \in(0, \infty)$.

Speculations about the above extensions of Müntz's Theorem are probably as old as Müntz's Theorem itself.

Somorjai [26] and Bak and Newman [2], [20] proved that

$$
R(\Lambda):=\{p / q: p, q \in M(\Lambda)\}
$$

is always dense in $C[0,1]$. (Though (1.1) is assumed throughout this paper, the above result holds for an arbitrary $\Lambda:=\left(\lambda_{i}\right)_{i=0}^{\infty}$ containing infinitely many distinct real numbers.) This surprising result says that while the set $M(\Lambda)$ of Müntz polynomials may be far from dense, the set $R(\Lambda)$ of Müntz rationals is always dense in $C[0,1]$, no matter what the underlying sequence $\Lambda$. In light of this result, Newman, in 1978 [20, p. 50], raises "the very sane, if very prosaic question": Are the functions

$$
\prod_{j=1}^{k}\left(\sum_{i=0}^{n_{j}} a_{i, j} x^{i^{2}}\right), \quad a_{i, j} \in \mathbb{R}, \quad n_{j} \in \mathbb{N},
$$

dense in $C[0,1]$ for some fixed $k \geq 2$ ? In other words does the "extra multiplication" have the same power that the "extra division" has in the Bak-NewmanSomorjai result? Newman speculated that it did not.

Denote the set of the above products by $H_{k}$. Since every natural number is the sum of four squares, $H_{4}$ contains all the monomials $x^{n}, n=0,1,2, \ldots$. However, $H_{k}$ is not a linear space, so Müntz's Theorem itself cannot be applied to resolve the denseness or non-denseness of $H_{4}$ in $C[0,1]$.

Section 7 of this paper deals with products of Müntz spaces and, in particular, answers the above question of Newman in the negative. For

$$
\Lambda_{j}:=\left(\lambda_{i, j}\right)_{i=0}^{\infty}, \quad 0=\lambda_{0, j}<\lambda_{1, j}<\lambda_{2, j}<\cdots, \quad j=1,2, \ldots,
$$

we define the sets

$$
M\left(\Lambda_{1}, \Lambda_{2}, \ldots, \Lambda_{k}\right):=\left\{p=\prod_{j=1}^{k} p_{j}: p_{j} \in M\left(\Lambda_{j}\right)\right\} .
$$

Bounded Remez-, Bernstein-, and Nikolskii-type inequalities are established for $M\left(\Lambda_{1}, \Lambda_{2}, \ldots, \Lambda_{k}\right)$ in the case that

$$
\sum_{i=1}^{\infty} \frac{1}{\lambda_{i, j}}<\infty, \quad j=1,2, \ldots, k .
$$

Any of these obviously implies that if (1.2) and (1.3) hold and $A \subset[0, \infty)$ is a compact set of positive Lebesgue measure, then $M\left(\Lambda_{1}, \Lambda_{2}, \ldots, \Lambda_{k}\right)$ is not dense in 
$C(A)$. In particular, $H_{4}$ is not dense in $C[0,1]$, which answers Newman's problem negatively. In addition, under the assumptions (1.2) and (1.3), our methods give an "almost characterization" of the uniform closure of $M\left(\Lambda_{1}, \Lambda_{2}, \ldots, \Lambda_{k}\right)$ on $A$ in terms of analyticity properties. This will likely be discussed in a later publication of the authors.

The results of Sections 6 and 7 can be proved fairly simply, once one has established the bounded Remez-type inequality of Section 5 for non-dense Müntz spaces $M(\Lambda)$. This is the central result of the paper, and is a result we believe should be a basic tool for dealing with problems about Müntz spaces, in addition to those discussed in Sections 6 and 7.

Let $\mathcal{P}_{n}$ denote the set of all algebraic polynomials of degree at most $n$ with real coefficients. For a fixed $s \in(0,1)$ let

$$
\mathcal{P}_{n}(s):=\left\{p \in \mathcal{P}_{n}: m(\{x \in[0,1]:|p(x)| \leq 1\}) \geq s\right\},
$$

where $m(\cdot)$ denotes the linear Lebesgue measure. The classical Remez inequality concerns the problem of bounding the uniform norm of a polynomial $p \in \mathcal{P}_{n}$ on $[0,1]$ given that its modulus is bounded by 1 on a subset of $[0,1]$ of Lebesgue measure at least $s$. That is, how large can $\|p\|_{[0,1]}$ (the uniform norm of $p$ on $[0,1]$ ) be if $p \in \mathcal{P}_{n}(s)$ ? The answer is given in terms of the Chebyshev polynomials. The extremal polynomials for the above problem are the Chebyshev polynomials $\pm T_{n}(x):= \pm \cos (n \arccos h(x))$, where $h$ is a linear function which scales $[0, s]$ or $[1-s, 1]$ onto $[-1,1]$.

For various proofs, extensions, and applications, see [13], [14], [15], [22], [23].

We generalize the Remez inequality in the following way. Let

$$
M_{n}(\Lambda):=\operatorname{span}\left\{x^{\lambda_{0}}, x^{\lambda_{1}}, \ldots, x^{\lambda_{n}}\right\} .
$$

That is, $M_{n}(\Lambda)$ is the collection of Müntz polynomials

$$
p(x):=\sum_{i=0}^{n} a_{i} x^{\lambda_{i}}, \quad a_{i} \in \mathbb{R} .
$$

We seek to find

$$
\max \left\{\frac{|p(0)|}{\|p\|_{A}}: 0 \neq p \in M_{n}(\Lambda), \quad A \subset[0,1], m(A) \geq s\right\}
$$

and

$$
\max \left\{\frac{|p(1)|}{\|p\|_{A}}: 0 \neq p \in M_{n}(\Lambda), \quad A \subset[0,1], m(A) \geq s\right\} .
$$

These two problems are no longer equivalent as they are in the polynomial case (since $x \rightarrow 1-x$ does not preserve membership in $M_{n}(\Lambda)$ ) and they have different answers. However, these two problems can be handled in essentially the same way. In Section 5 we concentrate on problem (1). Lemma 5.4 shows that an extremal function for problem (1) is the (generalized) Chebyshev polynomial

$$
T_{n}:=T_{n}\left\{\lambda_{0}, \lambda_{1}, \ldots, \lambda_{n} ;[1-s, 1]\right\}
$$

for $M_{n}(\Lambda)$ on $[1-s, 1]$ defined in Section 2. This reduces problem (1) to the interval case, $A=[1-s, 1]$. The interval case can then be handled by a bounded Chebyshev-type inequality

$$
\|p\|_{[0,1]} \leq c\|p\|_{[1-s, 1]}, \quad p \in M(\Lambda)=\operatorname{span}\left\{x^{\lambda_{0}}, x^{\lambda_{1}}, \ldots\right\},
$$


established in Section 3 for every $\Lambda:=\left(\lambda_{i}\right)_{i=0}^{\infty}$ satisfying $\sum_{i=1}^{\infty} 1 / \lambda_{i}<\infty$, where the constant $c$ depends only on $\Lambda$ and $s$ (and not on the "length" of $p$ ). This we first prove under the gap condition

$$
\inf \left\{\lambda_{i+1}-\lambda_{i}: i \in \mathbb{N}\right\}>0 .
$$

However, some comparison lemmas of Section 4 will allow us to drop this condition. This leads to the central result of the paper, a bounded Remez-type inequality for non-dense Müntz spaces (see Theorem 5.1) which states the following. For every $\Lambda:=\left(\lambda_{i}\right)_{i=0}^{\infty}$ with $\sum_{i=1}^{\infty} 1 / \lambda_{i}<\infty$, and for every $s>0$, there exists a constant $c$ depending only on $\Lambda$ and $s$ (and not on $\varrho$, $A$, or the "length" of $p$ ) so that

$$
\|p\|_{[0, \varrho]} \leq c\|p\|_{A}
$$

for every $p \in M(\Lambda)=\operatorname{span}\left\{x^{\lambda_{0}}, x^{\lambda_{1}}, \ldots\right\}$ and for every set $A \subset[\varrho, 1]$ of Lebesgue measure at least $s$.

One might note that the existence of such a bounded Remez-type inequality for a Müntz space $M(\Lambda)$ is equivalent to the non-denseness of $M(\Lambda)$ in $C[0,1]$. Indeed, if $M(\Lambda)$ is not dense in $C[0,1]$, then by Müntz's Theorem, $\sum_{i=1}^{\infty} 1 / \lambda_{i}<\infty$, and hence Theorem 5.1 implies that the above Remez-type inequality holds for $M(\Lambda)$. On the other hand, if $M(\Lambda)$ is dense in $C[0,1]$, then

$$
\sup _{p \in M(\Lambda)} \frac{|p(0)|}{\|p\|_{[1-s, 1]}}=\infty .
$$

This follows from

$$
\sup _{p \in C[0,1]} \frac{|p(0)|}{\|p\|_{[1-s, 1]}}=\infty
$$

by approximation.

In [8] the above result is established for lacunary Müntz spaces, that is, for $M(\Lambda)$ with

$$
\inf \left\{\lambda_{i+1} / \lambda_{i}: i \in \mathbb{N}\right\}>1 .
$$

Yet another remarkable consequence of the bounded Remez-type inequality of Theorem 5.1 is that the pointwise and locally uniform convergence of a sequence $\left(p_{i}\right)_{i=1}^{\infty} \subset M(\Lambda)$ on $[0, b)$ are equivalent whenever $\sum_{i=1}^{\infty} 1 / \lambda_{i}<\infty$. See Theorem 6.3. In fact, one can characterize the non-dense Müntz spaces within the Müntz spaces $M(\Lambda)$ as exactly those in which locally uniform and pointwise convergence on $[0, b)$ are equivalent.

\section{Notation}

The notations

$$
\begin{aligned}
\|p\|_{A} & :=\sup _{x \in A}|p(x)|, \\
\|p\|_{L_{w}^{q}(A)} & :=\left(\int_{A}|p|^{q} w\right)^{1 / q},
\end{aligned}
$$

and

$$
\|p\|_{L^{q}(A)}:=\left(\int_{A}|p|^{q}\right)^{1 / q}
$$


are used throughout this paper for measurable functions $p$ defined on a measurable set $A \subset[0, \infty)$, for non-negative measurable weight functions $w$ defined on $A$, and for $q \in(0, \infty)$. The space of all real-valued continuous functions on a set $A \subset[0, \infty)$ equipped with the uniform norm is denoted by $C(A)$.

The space $L_{w}^{q}(A)$ is defined as the collection of equivalence classes of real-valued measurable functions for which $\|f\|_{\left.L_{w}^{q}(A)\right)}<\infty$. The equivalence classes are defined by the equivalence relation $f \sim g$ if $f w=g w$ almost everywhere on $A$. When $A:=$ $[a, b]$ is a finite closed interval, we use the notation $L_{w}^{q}[a, b]:=L_{w}^{q}(A)$. When $w:=1$, we use the notation $L^{q}[a, b]:=L_{w}^{q}[a, b]$. Again, it is always our understanding that the space $L_{w}^{q}(A)$ is equipped with the $L_{w}^{q}(A)$ norm.

Throughout this paper $\Lambda:=\left(\lambda_{i}\right)_{i=0}^{\infty}$ denotes a sequence of real numbers satisfying

$$
0=\lambda_{0}<\lambda_{1}<\lambda_{2}<\cdots
$$

The non-negative-valued functions $x^{\lambda_{i}}$ are well-defined on $[0, \infty)$. The system

$$
\left(x^{\lambda_{0}}, x^{\lambda_{1}}, \ldots, x^{\lambda_{n}}\right)
$$

is called a (finite) Müntz system. The linear space

$$
M_{n}(\Lambda):=\operatorname{span}\left\{x^{\lambda_{0}}, x^{\lambda_{1}}, \ldots, x^{\lambda_{n}}\right\}
$$

over $\mathbb{R}$ is called a (finite) Müntz space. That is, the Müntz space $M_{n}(\Lambda)$ is the collection of Müntz polynomials

$$
p(x)=\sum_{i=0}^{n} a_{i} x^{\lambda_{i}}, \quad a_{i} \in \mathbb{R} .
$$

The set

$$
M(\Lambda):=\bigcup_{n=0}^{\infty} M_{n}(\Lambda)=\operatorname{span}\left\{x^{\lambda_{0}}, x^{\lambda_{1}}, \ldots\right\}
$$

is called the (infinite) Müntz space associated with $\Lambda$.

One of the most basic properties of a Müntz space $M_{n}(\Lambda)$ is the fact that it is a Chebyshev space on every $A \subset[0, \infty)$ containing at least $n+1$ points. That is, $M(\Lambda) \subset C(A)$ and every $p \in M_{n}(\Lambda)$ having at least $n+1$ (distinct) zeros in $A$ is identically 0 . In fact, Müntz spaces are the "canonical" examples for Chebyshev spaces and the following properties of Müntz spaces $M_{n}(\Lambda)$ are well known (see, for example, [9], [11], [21]).

Theorem 2.1 (Unique Interpolation Property). For every

$$
0 \leq x_{0}<x_{1}<\cdots<x_{n} \quad \text { and } \quad y_{0}, y_{1}, \ldots, y_{n} \in \mathbb{R}
$$

there exists a unique $p \in M_{n}(\Lambda)$ so that

$$
p\left(x_{j}\right)=y_{j}, \quad j=0,1, \ldots, n .
$$

Theorem 2.2 (Existence of Chebyshev Polynomials). Let $A$ be a compact subset of $[0, \infty)$ containing at least $n+1$ points. Then there exists a unique (extended) Chebyshev polynomial

$$
T_{n}:=T_{n}\left\{\lambda_{0}, \lambda_{1}, \ldots, \lambda_{n} ; A\right\}
$$


for $M_{n}(\Lambda)$ on $A$ defined by

$$
T_{n}(x)=c\left(x^{\lambda_{n}}-\sum_{i=0}^{n-1} a_{i} x^{\lambda_{i}}\right),
$$

where the numbers $a_{0}, a_{1}, \ldots, a_{n-1} \in \mathbb{R}$ are chosen to minimize

$$
\left\|x^{\lambda_{n}}-\sum_{i=0}^{n-1} a_{i} x^{\lambda_{i}}\right\|_{A},
$$

and where $c \in \mathbb{R}$ is a normalization constant chosen so that

$$
\left\|T_{n}\right\|_{A}=1
$$

and the sign of $c$ is determined by

$$
T_{n}(\max A)>0 .
$$

Theorem 2.3 (Alternation Characterization). The Chebyshev polynomial

$$
T_{n}:=T_{n}\left\{\lambda_{0}, \lambda_{1}, \ldots, \lambda_{n} ; A\right\} \in M_{n}(\Lambda)
$$

is uniquely characterized by the existence of an alternation set

$$
\left\{x_{0}<x_{1}<\cdots<x_{n}\right\} \subset A
$$

for which

$$
T_{n}\left(x_{j}\right)=(-1)^{n-j}=(-1)^{n-j}\left\|T_{n}\right\|_{A}, \quad j=0,1, \ldots, n .
$$

3. Bounded Chebyshev and Bernstein type inequalities for $M(\Lambda)$

The main results of this section are the following two theorems.

Theorem 3.1. Suppose $\sum_{i=1}^{\infty} 1 / \lambda_{i}<\infty$. Let $s \in(0,1)$. Then there exists a constant $c$ depending only on $\Lambda:=\left\{\lambda_{i}\right\}_{i=0}^{\infty}$ and $s$ (and not on the "length" of $p$ ) so that

$$
\|p\|_{[0,1]} \leq c\|p\|_{[1-s, 1]}
$$

for every $p \in M(\Lambda):=\operatorname{span}\left\{x^{\lambda_{0}}, x^{\lambda_{1}}, \ldots\right\}$.

Theorem 3.2. Suppose $\sum_{i=1}^{\infty} 1 / \lambda_{i}<\infty$ and $\lambda_{1} \geq 1$. Let $\varepsilon \in(0,1)$. Then there exists a constant $c$ depending only on $\Lambda:=\left(\lambda_{i}\right)_{i=0}^{\infty}$ and $\varepsilon$ (and not on the "length" of $p$ ) so that

$$
\left\|p^{\prime}\right\|_{[0,1-\varepsilon]} \leq c\|p\|_{[0,1]}
$$

for every $p \in M(\Lambda):=\operatorname{span}\left\{x^{\lambda_{0}}, x^{\lambda_{1}}, \ldots\right\}$.

To prove the above two theorems we need three lemmas. Lemmas 3.3 and 3.4 establish the conclusion of Theorems 3.2 and 3.1, respectively, under the gap condition

$$
\inf \left\{\lambda_{i+1}-\lambda_{i}: i \in \mathbb{N}\right\}>0,
$$

which is then dropped with the aid of Lemma 4.5. 
Lemma 3.3. Suppose $\sum_{i=1}^{\infty} 1 / \lambda_{i}<\infty, \lambda_{1} \geq 1$, and the gap condition (3.1) holds. Let $\varepsilon \in(0,1)$. Then there exists a constant $c$ depending only on $\Lambda:=\left(\lambda_{i}\right)_{i=0}^{\infty}$ and $\varepsilon$ (and not on the "length" of $p$ ) so that

$$
\left\|p^{\prime}\right\|_{[0,1-\varepsilon]} \leq c\|p\|_{[0,1]}
$$

for every $p \in M(\Lambda):=\operatorname{span}\left\{x^{\lambda_{0}}, x^{\lambda_{1}}, \ldots\right\}$.

Proof. Clarkson and Erdős [12] observed that under the conditions of the lemma, there exists a constant $c_{1}(\varepsilon)$ depending only on $\Lambda=\left(\lambda_{i}\right)_{i=0}^{\infty}$ and $\varepsilon \in(0,1)$ (and not on $i$ and $n$ ) so that

$$
\begin{aligned}
\left|a_{i}\right| & \leq c_{1}(\varepsilon)(1+\varepsilon)^{\lambda_{i}}\|p\|_{L^{2}[0,1]} \\
& \leq c_{1}(\varepsilon)(1+\varepsilon)^{\lambda_{i}}\|p\|_{[0,1]}
\end{aligned}
$$

for every $p \in M(\Lambda)$ of the form

$$
p(x)=\sum_{i=0}^{n} a_{i} x^{\lambda_{i}}, \quad a_{i} \in \mathbb{R} .
$$

Therefore, if $y \in[0,1-\varepsilon]$ and $\|p\|_{[0,1]} \leq 1$, then

$$
\begin{aligned}
\left|p^{\prime}(y)\right| & =\left|\sum_{i=1}^{n} \lambda_{i} a_{i} y^{\lambda_{i}-1}\right| \leq \sum_{i=1}^{n}\left|a_{i}\right| \lambda_{i}|y|^{\lambda_{i}-1} \\
& \leq \sum_{i=1}^{\infty} c_{1}(\varepsilon) \lambda_{i}(1+\varepsilon)^{\lambda_{i}}(1-\varepsilon)^{\lambda_{i}-1} \\
& =\frac{c_{1}(\varepsilon)}{1-\varepsilon} \sum_{i=1}^{\infty} \lambda_{i}\left(1-\varepsilon^{2}\right)^{\lambda_{i}} \\
& \leq \frac{c_{1}(\varepsilon)}{1-\varepsilon} \sum_{i=1}^{k} \lambda_{i}\left(1-\varepsilon^{2}\right)^{\lambda_{i}}+\sum_{i=k+1}^{\infty} i^{-2}=: c,
\end{aligned}
$$

where $k \in \mathbb{N}$ is chosen so that

$$
\left(1-\varepsilon^{2}\right)^{\lambda_{i}} \leq \frac{1}{\lambda_{i} i^{2}}, \quad i=k+1, k+2, \ldots
$$

This proves the lemma.

Lemma 3.4. Suppose $\sum_{i=1}^{\infty} 1 / \lambda_{i}<\infty$ and the gap condition (3.1) holds. Let $s \in(0,1)$. Then there exists a constant $c$ depending only on $\Lambda:=\left\{\lambda_{i}\right\}_{i=0}^{\infty}$ and $s$ (and not on the "length" of $p$ ) so that

$$
\|p\|_{[0,1]} \leq c\|p\|_{[1-s, 1]}
$$

for every $p \in M(\Lambda):=\operatorname{span}\left\{x^{\lambda_{0}}, x^{\lambda_{1}}, \ldots\right\}$.

Proof. Using the scaling $x \rightarrow x^{1 / \lambda_{1}}$, without loss of generality we may assume that $\lambda_{1}=1$. Suppose there exist

$$
p_{i} \in M(\Lambda), \quad i=1,2, \ldots,
$$

so that

$$
A_{i}:=\left\|p_{i}\right\|_{[0,1]} \rightarrow \infty,
$$


while

$$
\left\|p_{i}\right\|_{[1-s, 1]}=1, \quad i=1,2, \ldots
$$

Let $q_{i}:=p_{i} / A_{i}$. Note that $\left\|q_{i}\right\|_{[0,1]}=1$ and

$$
\lim _{i \rightarrow \infty}\left\|q_{i}\right\|_{[1-s, 1]}=0 .
$$

By Lemma 3.3, there exists a constant $c$ depending only on $\Lambda=\left(\lambda_{i}\right)_{i=0}^{\infty}$ and $\varepsilon$ so that

$$
\left\|q_{i}^{\prime}\right\|_{[0,1-\varepsilon]} \leq c\left\|q_{i}\right\|_{[0,1]}=c
$$

for every $\varepsilon \in(0,1)$. Hence $\left(q_{i}\right)_{i=1}^{\infty}$ is a sequence of uniformly bounded and equicontinuous functions on every closed subinterval of $[0,1)$. So, by the Arzela-Ascoli Theorem, we may extract a uniformly convergent subsequence on $[0,1-s / 2]$. By a theorem of Clarkson and Erdős [12], this subsequence converges uniformly to a function $F$ analytic on $(0,1-s / 2)$. Combining this with (3.2) and the Unicity Theorem, we can deduce that $F$ is identically zero. This is a contradiction since $\left\|q_{i}\right\|_{[0,1]}=1$ and

$$
\left\|q_{i}\right\|_{[0,1-s]}=\left\|q_{i}\right\|_{[0,1]}
$$

for every sufficiently large $i$. The lemma is now proved.

Proof of Theorem 3.1. Observe that $\lim _{i \rightarrow \infty} \lambda_{i} / i=\infty$. Therefore, there is an $m \in \mathbb{N}$ so that $\lambda_{i}>2 i$ whenever $i>m$. Let $\Gamma:=\left(\gamma_{i}\right)_{i=1}^{\infty}$ be defined by

$$
\gamma_{i}:= \begin{cases}\min \left\{\lambda_{i}, i\right\} & \text { if } i=0,1, \ldots, m, \\ \lambda_{i} / 2+i & \text { if } i=m+1, m+2, \ldots .\end{cases}
$$

Then $0=\gamma_{0}<\gamma_{1}<\gamma_{2}<\cdots, \sum_{i=1}^{\infty} 1 / \gamma_{i}<\infty$,

$$
\gamma_{i} \leq \lambda_{i}, \quad i=0,1,2, \ldots
$$

and

$$
\inf \left\{\gamma_{i+1}-\gamma_{i}: i \in \mathbb{N}\right\}>0 .
$$

Now Lemma 3.4 and the first part of Lemma 4.5 will yield the theorem.

Proof of Theorem 3.2. Without loss of generality we may assume that $\lambda_{1}=1$. Let $\Gamma:=\left(\gamma_{i}\right)_{i=0}^{\infty}$ be defined as in the proof of Theorem 3.1. Now Lemma 3.3, Theorem 3.1 , and the second part of Lemma 4.5 yield the theorem.

\section{Comparison lemmas}

One of the basic properties of a Müntz system

$$
\left(x^{\lambda_{0}}, x^{\lambda_{1}}, \ldots, x^{\lambda_{n}}\right), \quad 0=\lambda_{0}<\lambda_{1}<\cdots<\lambda_{n},
$$

is that it is a Descartes system on every interval $[a, b] \subset(0, \infty)$; see [21]. The following comparison lemma, due to Pinkus and Smith [25], is valid for every Descartes system. 
Lemma 4.1. Suppose $\left(f_{0}, f_{1}, \ldots, f_{n}\right)$ is a Descartes system on $[a, b]$. Suppose

$$
\begin{array}{ll}
p=f_{\alpha}+\sum_{i=1}^{k} a_{i} f_{\lambda_{i}}, & a_{i} \in \mathbb{R}, \\
q=f_{\alpha}+\sum_{i=1}^{k} b_{i} f_{\gamma_{i}}, & b_{i} \in \mathbb{R},
\end{array}
$$

where $0 \leq \lambda_{1}<\lambda_{2}<\cdots<\lambda_{k} \leq n, 0 \leq \gamma_{1}<\gamma_{2}<\cdots<\gamma_{k} \leq n$,

$$
0 \leq \gamma_{i} \leq \lambda_{i}<\alpha, \quad i=1,2, \ldots, m,
$$

and

$$
\alpha<\lambda_{i} \leq \gamma_{i} \leq n, \quad i=m+1, m+2, \ldots, k,
$$

with strict inequality for at least one index $i=1,2, \ldots, k$. Then

$$
p\left(x_{i}\right)=q\left(x_{i}\right)=0, \quad i=1,2, \ldots, k,
$$

with distinct $x_{i} \in[a, b]$ implies

$$
|p(x)| \leq|q(x)|
$$

for every $x \in[a, b]$ with strict inequality for every

$$
x \in[a, b] \backslash\left\{x_{1}, x_{2}, \ldots, x_{k}\right\} .
$$

To formulate the next lemmas we introduce the following notation. Let

$$
0=\lambda_{0}<\lambda_{1}<\cdots<\lambda_{n}, \quad 0=\gamma_{0}<\gamma_{1}<\cdots<\gamma_{n},
$$

and

$$
\gamma_{i} \leq \lambda_{i}, \quad i=1,2, \ldots, n .
$$

Let

$$
M_{n}(\Lambda):=\operatorname{span}\left\{x^{\lambda_{0}}, x^{\lambda_{1}}, \ldots, x^{\lambda_{n}}\right\}
$$

and

$$
M_{n}(\Gamma):=\operatorname{span}\left\{x^{\gamma_{0}}, x^{\gamma_{1}}, \ldots, x^{\gamma_{n}}\right\} .
$$

Let $s \in(0,1)$ be fixed. Let

$$
T_{n, \lambda}:=T_{n}\left\{\lambda_{0}, \lambda_{1}, \ldots, \lambda_{n} ;[1-s, 1]\right\}
$$

and

$$
T_{n, \gamma}:=T_{n}\left\{\gamma_{0}, \gamma_{1}, \ldots, \gamma_{n} ;[1-s, 1]\right\}
$$

denote the Chebyshev polynomials on $[1-s, 1]$ for $M_{n}(\Lambda)$ and $M_{n}(\Gamma)$, respectively (see Theorem 2.2).

Lemma 4.2. Let $y \in[0,1-s)$ be fixed. Then both

$$
\max _{0 \neq p \in M_{n}(\Lambda)} \frac{|p(y)|}{\|p\|_{[1-s, 1]}}
$$

and

$$
\max _{0 \neq p \in M_{n}(\Lambda)} \frac{\left|p^{\prime}(y)\right|}{\|p\|_{[1-s, 1]}}
$$

are attained by $p=T_{n, \lambda}$. In the second case we assume $\lambda_{1} \geq 1$ if $y=0$. 
Proof. A simple compactness argument shows that the maxima in the lemma are attained by some $p^{*} \in M_{n}(\Lambda)$ and $q^{*} \in M_{n}(\Lambda)$, which can be identified as $T_{n, \lambda}$ by a standard variational method. See for example [16, p. 295], [23, p. 101] where arguments of this variety are given.

Lemma 4.3. We have

$$
\left|T_{n, \lambda}(0)\right| \leq\left|T_{n, \gamma}(0)\right|
$$

Further, if $\lambda_{1}=\gamma_{1}=1$, then

$$
\left|T_{n, \lambda}^{\prime}(0)\right| \leq\left|T_{n, \gamma}^{\prime}(0)\right| .
$$

Proof. Let $p \in M_{n}(\Gamma)$ interpolate $T_{n, \lambda}$ at the $n$ zeros of $T_{n, \lambda}$ in $[1-s, 1]$ and at 0 . It follows from Lemma 4.1 that

$$
|p(x)| \leq\left|T_{n, \lambda}(x)\right|, \quad x \in[0,1] .
$$

In particular,

$$
\|p\|_{[1-s, 1]} \leq\left\|T_{n, \lambda}\right\|_{[1-s, 1]}=1,
$$

which, together with $p(0)=T_{n, \lambda}(0)$ and Lemma 4.2 , gives

$$
\left|T_{n, \lambda}(0)\right|=|p(0)| \leq \frac{|p(0)|}{\|p\|_{[1-s, 1]}} \leq \frac{\left|T_{n, \gamma}(0)\right|}{\left\|T_{n, \gamma}\right\|_{[1-s, 1]}}=\left|T_{n, \gamma}(0)\right| .
$$

This proves the first part of the lemma.

The second part of the lemma can be proved in essentially the same way. Let $0 \neq p \in M_{n}(\Gamma)$ interpolate $T_{n, \lambda}$ at the $n$ zeros of $T_{n, \lambda}$ in $[1-s, 1]$. Note that $p^{\prime}(0) \neq 0$, otherwise

$$
p \in \operatorname{span}\left\{x^{\gamma_{j}}: j=0,1, \ldots, n, j \neq 1\right\}
$$

cannot have $n$ zeros in $[1-s, 1]$. Similarly $T_{n, \lambda}^{\prime}(0) \neq 0$. Normalize $p$ so that

$$
p^{\prime}(0)=T_{n, \lambda}^{\prime}(0) \text {. }
$$

It follows from Lemma 4.1 that

$$
|p(x)| \leq\left|T_{n, \lambda}(x)\right|, \quad x \in[0,1] .
$$

In particular

$$
\|p\|_{[1-s, 1]} \leq\left\|T_{n, \lambda}\right\|_{[1-s, 1]}=1,
$$

which, together with $p^{\prime}(0)=T_{n, \lambda}^{\prime}(0)$ and Lemma 4.2, yields

$$
\left|T_{n, \lambda}^{\prime}(0)\right|=\left|p^{\prime}(0)\right| \leq \frac{\left|p^{\prime}(0)\right|}{\|p\|_{[1-s, 1]}} \leq \frac{\left|T_{n, \gamma}^{\prime}(0)\right|}{\left\|T_{n, \gamma}\right\|_{[1-s, 1]}}=\left|T_{n, \gamma}^{\prime}(0)\right| .
$$

This proves the second part of the lemma.

Lemma 4.4. The functions $\left|T_{n, \lambda}\right|$ and $\left|T_{n, \gamma}\right|$ are decreasing on $[0,1-s]$. Further, if $\lambda_{1}=\gamma_{1}=1$, then $\left|T_{n, \lambda}^{\prime}\right|$ and $\left|T_{n, \gamma}^{\prime}\right|$ are also decreasing on $[0,1-s]$.

Proof. Suppose $\left|T_{n, \lambda}\right|$ is not monotone decreasing on $[0,1-s]$. Then

$$
T_{n, \lambda}^{\prime} \in \operatorname{span}\left\{x^{\lambda_{1}-1}, x^{\lambda_{2}-1}, \ldots, x^{\lambda_{n}-1}\right\}
$$

must have at least $n$ zeros in $(0,1)$, which is impossible. This proves the first statement of the lemma. 
Suppose now $\lambda_{1}=1$ and $\left|T_{n, \lambda}^{\prime}\right|$ is not monotone decreasing on $[0,1-s]$. Then

$$
T_{n, \lambda}^{\prime \prime} \in \operatorname{span}\left\{x^{\lambda_{2}-2}, x^{\lambda_{3}-2}, \ldots, x^{\lambda_{n}-2}\right\}
$$

must have at least $n-1$ zeros in $(0,1)$, which is impossible. This proves the second statement of the lemma.

The main result of this section is the following lemma. It plays a crucial role in the proof of Theorems 3.1 and 3.2 of the previous section.

Lemma 4.5. We have

$$
\max _{0 \neq p \in M_{n}(\Lambda)} \frac{\|p\|_{[0,1]}}{\|p\|_{[1-s, 1]}} \leq \max _{0 \neq p \in M_{n}(\Gamma)} \frac{\|p\|_{[0,1]}}{\|p\|_{[1-s, 1]}} .
$$

Further, if $\lambda_{1}=\gamma_{1}=1$, then

$$
\max _{0 \neq p \in M_{n}(\Lambda)} \frac{\left\|p^{\prime}\right\|_{[0,1-s]}}{\|p\|_{[1-s, 1]}} \leq \max _{0 \neq p \in M_{n}(\Gamma)} \frac{\left\|p^{\prime}\right\|_{[0,1-s]}}{\|p\|_{[1-s, 1]}} .
$$

Proof. Combining Lemmas 4.2, 4.3, and 4.4, we obtain for every $y \in[0,1-s)$ that

$$
\begin{aligned}
\max _{0 \neq p \in M_{n}(\Lambda)} \frac{|p(y)|}{\|p\|_{[1-s, 1]}} & =\frac{\left|T_{n, \lambda}(y)\right|}{\left\|T_{n, \lambda}\right\|_{[1-s, 1]}}=\left|T_{n, \lambda}(y)\right| \leq\left|T_{n, \lambda}(0)\right| \leq\left|T_{n, \gamma}(0)\right| \\
& =\frac{\left|T_{n, \gamma}(0)\right|}{\left\|T_{n, \gamma}\right\|_{[1-s, 1]}} \leq \max _{0 \neq p \in M_{n}(\Gamma)} \frac{\|p\|_{[0,1-s]}}{\|p\|_{[1-s, 1]}}
\end{aligned}
$$

which implies the first inequality of the lemma.

Similarly, combining Lemmas $4.2,4.3$, and 4.4 , we obtain for every $y \in[0,1-s)$ that

$$
\begin{aligned}
\max _{0 \neq p \in M_{n}(\Lambda)} \frac{\left|p^{\prime}(y)\right|}{\|p\|_{[1-s, 1]}} & =\frac{\left|T_{n, \lambda}^{\prime}(y)\right|}{\left\|T_{n, \lambda}\right\|_{[1-s, 1]}}=\left|T_{n, \lambda}^{\prime}(y)\right| \leq\left|T_{n, \lambda}^{\prime}(0)\right| \leq\left|T_{n, \gamma}^{\prime}(0)\right| \\
& =\frac{\left|T_{n, \gamma}^{\prime}(0)\right|}{\left\|T_{n, \gamma}\right\|_{[1-s, 1]}} \leq \max _{0 \neq p \in M_{n}(\Gamma)} \frac{\left\|p^{\prime}\right\|_{[0,1-s]}}{\|p\|_{[1-s, 1]}},
\end{aligned}
$$

which implies the second inequality of the lemma.

\section{Bounded Remez-Type inequality for nON-DEnse MÜntZ SPACES}

The central result of this paper is the following.

Theorem 5.1. Suppose $\sum_{i=1}^{\infty} 1 / \lambda_{i}<\infty$. Let $s>0$. Then there exists a constant $c$ depending only on $\Lambda:=\left(\lambda_{i}\right)_{i=0}^{\infty}$ and $s$ (and not on $\varrho, A$, or the "length" of $p$ ) so that

$$
\|p\|_{[0, \varrho]} \leq c\|p\|_{A}
$$

for every $p \in M(\Lambda):=\operatorname{span}\left\{x^{\lambda_{0}}, x^{\lambda_{1}}, \ldots\right\}$ and for every set $A \subset[\varrho, 1]$ of Lebesgue measure at least $s$.

The proof of Theorem 5.1 is based on interpolation. By the Unique Interpolation Property of Chebyshev spaces, associated with

$$
0 \leq x_{0}<x_{1}<\cdots<x_{n}
$$

we can uniquely define

$$
\ell_{k}:=\ell_{k}\left\{x_{0}, x_{1}, \ldots, x_{n}\right\} \in M_{n}(\Lambda), \quad k=0,1, \ldots, n,
$$


so that

$$
\ell_{k}\left\{x_{0}, x_{1}, \ldots, x_{n}\right\}\left(x_{j}\right)=\delta_{j, k}:= \begin{cases}1 & \text { if } j=k \\ 0 & \text { if } j \neq k\end{cases}
$$

Lemma 5.2. Let

$$
0<x_{0}<x_{1}<\cdots<x_{n} \quad \text { and } \quad 0<\tilde{x}_{0}<\tilde{x}_{1}<\cdots<\tilde{x}_{n} .
$$

Suppose $0 \leq k \leq n$ and

$$
\begin{array}{ll}
x_{j} \leq \tilde{x}_{j} & \text { for } j=0,1, \ldots, k-1, \\
x_{j}=\tilde{x}_{j} & \text { for } j=k, \\
x_{j} \geq \tilde{x}_{j} & \text { for } j=k+1, k+2, \ldots, n .
\end{array}
$$

For the sake of brevity let

$$
\ell_{k}:=\ell_{k}\left\{x_{0}, x_{1}, \ldots, x_{n}\right\}
$$

and

$$
\tilde{\ell}_{k}:=\ell_{k}\left\{\tilde{x}_{0}, \tilde{x}_{1}, \ldots, \tilde{x}_{n}\right\}
$$

Then

$$
\left|\ell_{k}(0)\right| \leq\left|\tilde{\ell}_{k}(0)\right|
$$

Proof. It is sufficient to prove the lemma in the case that there is an index $m$ so that $0 \leq m \leq n, m \neq k$, and

$$
\begin{array}{ll}
x_{j}=\tilde{x}_{j} & \text { for } j=0,1, \ldots, n, \quad j \neq m, \\
x_{m}<\tilde{x}_{m} & \text { if } m<k, \\
x_{m}>\tilde{x}_{m} & \text { if } m>k .
\end{array}
$$

The general case of the lemma then follows from repeated applications of the above special cases. Note that in the above special cases

$$
\ell_{k}-\tilde{\ell}_{k} \in M_{n}(\Lambda)
$$

has a zero at each of the points

$$
x_{0}, x_{1}, \ldots, x_{m-1}, x_{m+1}, x_{m+2}, \ldots, x_{n},
$$

hence it changes sign at each of these points, and does not have any other zero in $[0, \infty)$. It is also obvious that

$$
\operatorname{sign}\left(\ell_{k}(x)\right)=\operatorname{sign}\left(\tilde{\ell}_{k}(x)\right), \quad x \in\left[0, x_{0}\right] .
$$

This, together with the previous observation and the inequality $x_{0} \leq \tilde{x}_{0}$, yields that

$$
\left|\ell_{k}(0)\right| \leq\left|\tilde{\ell}_{k}(0)\right|,
$$

otherwise $\ell_{k}-\tilde{\ell}_{k}$ would have a zero in $\left(x_{m-1}, x_{m+1}\right)$, where $x_{-1}:=0$ and $x_{n+1}:=\infty$, which is impossible.

By a simple scaling we can extend Lemma 5.2 as follows. We use the notation introduced in Lemma 5.2.

Lemma 5.3. Let

$$
0<x_{0}<x_{1}<\cdots<x_{n} \quad \text { and } \quad 0<\tilde{x}_{0}<\tilde{x}_{1}<\cdots<\tilde{x}_{n} .
$$

Suppose $0 \leq k \leq n, \alpha \geq 0$, and 
Then

$$
\begin{array}{ll}
x_{j} \leq \tilde{x}_{j}-\alpha & \text { for } j=0,1, \ldots, k-1, \\
x_{j}=\tilde{x}_{j}-\alpha & \text { for } j=k \\
x_{j} \geq \tilde{x}_{j}-\alpha & \text { for } j=k+1, k+2, \ldots, n .
\end{array}
$$

$$
\left|\ell_{k}(0)\right| \leq\left|\tilde{\ell}_{k}(0)\right| .
$$

Proof. If $\alpha=0$, then Lemma 5.2 yields the lemma. So we may suppose that $\alpha>0$. Let

$$
\begin{gathered}
\beta:=\frac{x_{k}}{\tilde{x}_{k}}=\frac{\tilde{x}_{k}-\alpha}{\tilde{x}_{k}}, \\
x_{j}^{*}:=\beta \tilde{x}_{j}, \quad j=0,1, \ldots, n,
\end{gathered}
$$

and

$$
\ell_{k}^{*}:=\ell_{k}\left\{x_{0}^{*}, x_{1}^{*}, \ldots, x_{n}^{*}\right\}, \quad k=0,1, \ldots, n
$$

Obviously

$$
\tilde{\ell}_{k}(\beta x)=\ell_{k}^{*}(x), \quad x \in[0, \infty),
$$

and

$$
\begin{array}{ll}
x_{j} \leq x_{j}^{*} & \text { for } j=0,1, \ldots, k-1, \\
x_{j}=x_{j}^{*} & \text { for } j=k, \\
x_{j} \geq x_{j} & \text { for } j=k+1, k+2, \ldots, n .
\end{array}
$$

Hence Lemma 5.2 implies that

$$
\left|\ell_{k}(0)\right| \leq\left|\ell_{k}^{*}(0)\right|=\left|\tilde{\ell}_{k}(0)\right|,
$$

which finishes the proof.

The next two lemmas are interesting for their own right. They show that the appropriately placed Chebyshev polynomial is always extremal for the Remez-type inequality we are considering.

Lemma 5.4. Let $A \subset[0,1]$ be a closed set of Lebesgue measure at least $s \in(0,1)$. Then

$$
|p(0)| \leq\left|T_{n}\left\{\lambda_{0}, \lambda_{1}, \ldots, \lambda_{n} ;[1-s, 1]\right\}(0)\right| \cdot\|p\|_{A}
$$

for every $p \in M_{n}(\Lambda)$.

Proof. If $0 \in A$, then the statement is trivial. So assume that $0 \notin A$. Let

$$
\tilde{x}_{0}<\tilde{x}_{1}<\cdots<\tilde{x}_{n}
$$

denote the extreme points of

$$
T_{n}:=T_{n}\left\{\lambda_{0}, \lambda_{1}, \ldots, \lambda_{n} ;[1-s, 1]\right\}
$$

in $[1-s, 1]$, that is,

$$
T_{n}\left(\tilde{x}_{j}\right)=(-1)^{n-j}, \quad j=0,1, \ldots, n .
$$

Let $x_{j} \in A, j=0,1, \ldots, n$, be defined by

$$
m\left(\left[x_{j}, 1\right] \cap A\right)=m\left(\left[\tilde{x}_{j}, \tilde{x}_{n}\right]\right)=\tilde{x}_{n}-\tilde{x}_{j} .
$$


Since $A$ is a closed subset of $[0,1]$ with $m(A) \geq s$, such points $x_{j} \in A$ exist. Let $p \in M_{n}(\Lambda)$. Then, using Lemma 5.3 , we obtain

$$
\begin{aligned}
|p(0)| & =\left|\sum_{k=0}^{n} p\left(x_{k}\right) \ell_{k}(0)\right| \\
& \leq\left(\sum_{k=0}^{n}\left|\ell_{k}(0)\right|\right)\|p\|_{A} \\
& \leq\left(\sum_{k=0}^{n}\left|\tilde{\ell}_{k}(0)\right|\right)\|p\|_{A} \\
& =\left|\sum_{k=0}^{n}(-1)^{n-k} \tilde{\ell}_{k}(0)\right|\|p\|_{A} \\
& =\left|\sum_{k=0}^{n} T_{n}\left(\tilde{x}_{k}\right) \tilde{\ell}_{k}(0)\right|\|p\|_{A} \\
& =\left|T_{n}(0)\right| \cdot\|p\|_{A}
\end{aligned}
$$

and the lemma follows. In the rest of the proof we justify each line above.

Note that $p \in M_{n}(\Lambda)$ and $\sum_{k=0}^{n} p\left(x_{k}\right) \ell_{k} \in M_{n}(\Lambda)$ agree at $x_{0}, x_{1}, \ldots, x_{n}$. Since $M_{n}(\Lambda)$ is a Chebyshev space of dimension $n+1$ on $[0,1]$, we can deduce that $p=\sum_{k=0}^{n} p\left(x_{k}\right) \ell_{k}$, and the first line above follows by substituting 0 . The second line follows by the triangle inequality. Note that $x_{k} \in A$, so $\left|p\left(x_{k}\right)\right| \leq\|p\|_{A}$ for each $k$. To see the third line we need the inequalities $\left|\ell_{k}(0)\right| \leq\left|\tilde{\ell}_{k}(0)\right|$ for each $k$. These follow from Lemma 5.3. The assumptions of Lemma 5.3 are satisfied since the construction obviously implies the inequalities $0<x_{j} \leq \tilde{x}_{j}$ and $\tilde{x}_{i}-\tilde{x}_{j} \leq x_{i}-x_{j}$ for every $0 \leq j<i \leq n$. The fourth line follows from the observation that $\tilde{\ell}_{k}(0)=$ $-\tilde{\ell}_{k+1}(0)$ for each $k=0,1, \ldots, n-1$. This can be deduced from the fact that $\tilde{\ell}_{k}$ changes sign exactly at

$$
\tilde{x}_{1}, \tilde{x}_{2}, \ldots, \tilde{x}_{k-1}, \tilde{x}_{k+1}, \tilde{x}_{k+2}, \ldots, \tilde{x}_{n},
$$

while $\tilde{\ell}_{k}\left(\tilde{x}_{k}\right)=1$. The fifth line uses the fact that

$$
T_{n}\left(\tilde{x}_{k}\right)=(-1)^{n-k}, \quad k=0,1, \ldots, n .
$$

Finally, the last line follows by observing that $T_{n} \in M_{n}(\Lambda)$ and $\sum_{k=0}^{n} T_{n}\left(\tilde{x}_{k}\right) \tilde{\ell}_{k} \in$ $M_{n}(\Lambda)$ agree at $\tilde{x}_{0}, \tilde{x}_{1}, \ldots, \tilde{x}_{n}$. Since $M_{n}(\Lambda)$ is a Chebyshev space of dimension $n+1$ on $[0,1]$, we can deduce that $T_{n}=\sum_{k=0}^{n} T_{n}\left(\tilde{x}_{k}\right) \tilde{\ell}_{k}$, and on substituting 0 , we obtain the last line.

Lemma 5.5. Let $A$ be a closed subset of $[0,1]$ with Lebesgue measure at least $s \in$ $(0,1)$. Then

$$
|p(y)| \leq\left|T_{n}\left\{\lambda_{0}, \lambda_{1}, \ldots, \lambda_{n} ;[1-s, 1]\right\}(0)\right| \cdot\|p\|_{A}
$$

for every $p \in M_{n}(\Lambda)$ and $y \in[0, \inf A)$.

Proof. Let $y \in[0, \inf A)$ be fixed. Simple compactness and perturbation arguments show that

$$
\max _{0 \neq p \in M_{n}(\Lambda)} \frac{|p(y)|}{\|p\|_{A}}
$$


is attained by

$$
T_{n, A}:=T_{n}\left\{\lambda_{0}, \lambda_{1}, \ldots, \lambda_{n} ; A\right\} .
$$

Note that $\lambda_{0}=0$ implies that $T_{n, A}$ is decreasing on $[0, \inf A]$, otherwise

$$
T_{n, A}^{\prime} \in \operatorname{span}\left\{x^{\lambda_{1}-1}, x^{\lambda_{2}-1}, \ldots, x^{\lambda_{n}-1}\right\}
$$

must have at least $n+1$ zeros in $(0,1]$, which is impossible. Hence, by Lemma 5.4,

$$
\frac{|p(y)|}{\|p\|_{A}} \leq \frac{\left|T_{n, A}(y)\right|}{\left\|T_{n, A}\right\|_{A}}=\left|T_{n, A}(y)\right| \leq\left|T_{n, A}(0)\right| \leq\left|T_{n}\left\{\lambda_{0}, \lambda_{1}, \ldots, \lambda_{n} ;[1-s, 1]\right\}(0)\right|
$$

for every $0 \neq p \in M_{n}(\Lambda)$. This finishes the proof.

Proof of Theorem 5.1. Without loss of generality we may assume that $A$ is closed. Let

$$
T_{n}:=T_{n}\left\{\lambda_{0}, \lambda_{1}, \ldots, \lambda_{n} ;[1-s, 1]\right\} .
$$

By Theorem 3.1, there exists a constant $c$ depending only on $\Lambda:=\left(\lambda_{i}\right)_{i=0}^{\infty}$ and $s$ (and not on the "length" of $p$ ) so that

$$
\left\|T_{n}\right\|_{[0,1]} \leq c\left\|T_{n}\right\|_{[1-s, 1]}=c
$$

for every $p \in M(\Lambda):=\operatorname{span}\left\{x^{\lambda_{0}}, x^{\lambda_{1}}, \ldots\right\}$. By combining this with Lemma 5.5, there exists a constant $c$ depending only on $\Lambda:=\left(\lambda_{i}\right)_{i=0}^{\infty}$ and $s$ (and not on $\varrho, A$, or the "length" of $p$ ) so that

$$
\|p\|_{[0, \varrho]} \leq c\|p\|_{A}
$$

for every $p \in M(\Lambda):=\operatorname{span}\left\{x^{\lambda_{0}}, x^{\lambda_{1}}, \ldots\right\}$ and for every set $A \subset[\varrho, 1]$ of Lebesgue measure at least $s$.

The next theorem establishes an $L^{q}$ version of Theorem 5.1.

Theorem 5.6. Suppose $\sum_{i=1}^{\infty} 1 / \lambda_{i}<\infty$. Let $s>0$ and $q \in(0, \infty)$. Then there exists a constant $c$ depending only on $\Lambda:=\left(\lambda_{i}\right)_{i=0}^{\infty}, s$, and $q$ (and not on $\varrho$, $A$, or the "length" of p) so that

$$
\|p\|_{[0, \varrho]} \leq c\|p\|_{L^{q}(A)}
$$

for every $p \in M(\Lambda):=\operatorname{span}\left\{x^{\lambda_{0}}, x^{\lambda_{1}}, \ldots\right\}$ and for every set $A \subset[\varrho, 1]$ of Lebesgue measure at least $s$.

Proof. Note that $m(A) \geq s$ implies that

$$
m\left(\left\{x \in A:|p(x)| \geq\left(\frac{2}{s}\right)^{1 / q}\|p\|_{L^{q}(A)}\right\}\right) \leq \frac{s}{2},
$$

hence

$$
m\left(\left\{x \in A:|p(x)|<\left(\frac{2}{s}\right)^{1 / q}\|p\|_{L^{q}(A)}\right\}\right) \geq \frac{s}{2} .
$$

The theorem now follows from Theorem 5.1. 


\section{MÜntZ's ThEOREM ON COMPACT SETS OF POSITIVE MEASURE}

The results of this section are straightforward consequences of the Remez-type inequality of Theorem 5.1.

Theorem 6.1. Suppose $\sum_{i=1}^{\infty} 1 / \lambda_{i}<\infty$ and $A \subset[0, \infty)$ is a set of positive Lebesgue measure. Then $M(\Lambda)$ is not dense in $C(A)$.

Moreover, if the gap condition

$$
\inf \left\{\lambda_{i+1}-\lambda_{i}: i \in \mathbb{N}\right\}>0
$$

holds, then every function $f \in C(A)$ from the uniform closure of $M(\Lambda)$ on $A$ is of the form

$$
f(x)=\sum_{i=0}^{\infty} a_{i} x^{\lambda_{i}}, \quad x \in A \cap\left[0, r_{A}\right),
$$

where

$$
r_{A}:=\sup \{x \in[0, \infty): m(A \cap(x, \infty))>0\}
$$

is the essential supremum of $A$.

If the gap condition (6.1) does not hold, then every function $f \in C(A)$ from the uniform closure of $M(\Lambda)$ on $A$ can still be extended analytically throughout the region

$$
\left\{z \in \mathbb{C} \backslash(-\infty, 0]:|z|<r_{A}\right\} .
$$

Proof. Suppose $f \in C(A)$ and suppose there is a sequence $\left(p_{i}\right)_{i=1}^{\infty} \subset M(\Lambda)$ so that

$$
\lim _{i \rightarrow \infty}\left\|p_{i}-f\right\|_{A}=0 \text {. }
$$

Then the sequence $\left(p_{i}\right)_{i=1}^{\infty}$ is uniformly Cauchy on $A$. Therefore, Theorem 5.1 and the definition of $r_{A}$ yield that $\left(p_{i}\right)_{i=1}^{\infty}$ is uniformly Cauchy on every closed subinterval of $\left[0, r_{A}\right)$. If the gap condition (6.1) holds, then the characterization of the uniform closure of $M(\Lambda)$ on $A$ follows from the results of Clarkson and Erdös [12]. The result of Clarkson and Erdös [12] we need here claims that if the gap condition (6.1) holds and $0 \leq a<b<\infty$, then every function $f \in C[a, b]$ from the uniform closure of $M(\Lambda)$ on $[a, b]$ is of the form

$$
f(x)=\sum_{i=0}^{\infty} a_{i} x^{\lambda_{i}}, \quad x \in[a, b) .
$$

If the gap condition (6.1) does not hold, then results of Schwartz [24] yield the theorem. The result of Schwartz [24] we need here claims that for every $0 \leq a<$ $b<\infty$, even if the gap condition (6.1) does not hold, every function $f \in C[a, b]$ from the uniform closure of $M(\Lambda)$ on $[a, b]$ can still be extended analytically throughout the region

$$
\{z \in \mathbb{C} \backslash(-\infty, 0]:|z|<b\}
$$

Theorem 6.2. Suppose $A \subset[0, \infty)$ is a compact set of positive Lebesgue measure. Then $M(\Lambda)$ is dense in $C(A)$ if and only if $\sum_{i=1}^{\infty} 1 / \lambda_{i}=\infty$. 
Proof. Suppose $\sum_{i=1}^{\infty} 1 / \lambda_{i}=\infty$. Let $f \in C(A)$. By Tietze's Theorem there exists an $\tilde{f} \in C[0,1]$ so that $\tilde{f}(x)=f(x)$ for every $x \in A$. By Müntz's Theorem there is a sequence $\left(p_{i}\right)_{i=1}^{\infty} \subset M(\Lambda)$ so that

$$
\lim _{i \rightarrow \infty}\left\|\tilde{f}-p_{i}\right\|_{[0,1]}=0 .
$$

Therefore

$$
\lim _{i \rightarrow \infty}\left\|f-p_{i}\right\|_{A}=0,
$$

which finishes the trivial part of the theorem. Suppose now that $\sum_{i=1}^{\infty} 1 / \lambda_{i}<\infty$. Then Theorem 6.1 yields that $M(\Lambda)$ is not dense in $C(A)$.

The following surprising theorem shows that if $\sum_{i=1}^{\infty} 1 / \lambda_{i}<\infty$, then the pointwise and locally uniform convergence of a sequence $\left(p_{i}\right)_{i=1}^{\infty} \subset M(\Lambda)$ on $[0, b)$ are equivalent. An amusing consequence of this is that if $\sum_{i=1}^{\infty} 1 / \lambda_{i}<\infty$, then the set

$$
\left\{f: f(x)=\sum_{i=0}^{\infty} a_{i} x^{\lambda_{i}}, \quad a_{i} \in \mathbb{R}, \quad x \in[0, b)\right\}
$$

is closed under pointwise convergence.

Theorem 6.3. Suppose $\sum_{i=1}^{\infty} 1 / \lambda_{i}<\infty$. Let $A \subset[0, \infty)$ be a set of positive Lebesgue measure, and let $r_{A}$ be the essential supremum of $A$ defined as in Theorem 6.1. Assume $\left(p_{i}\right)_{i=1}^{\infty} \subset M(\Lambda)$ and

$$
p_{i}(x) \rightarrow f(x), \quad x \in A .
$$

Then $\left(p_{i}\right)_{i=1}^{\infty}$ converges uniformly on every closed subinterval of $\left[0, r_{A}\right)$.

This characterizes non-dense Müntz spaces within Müntz spaces, since in any Müntz space $M(\Lambda)$ with $\sum_{i=1}^{\infty} 1 / \lambda_{i}=\infty$ and for any $A \subset[0, \infty)$ of positive Lebesgue measure, there exists a sequence $\left(p_{i}\right)_{i=1}^{\infty} \subset M(\Lambda)$ that converges pointwise on $[0, \infty)$ but not locally uniformly on $A$. This follows easily from Müntz's Theorem.

Proof of Theorem 6.3. Let $\delta \in\left(0, r_{A}\right)$ be fixed. Egoroff's Theorem and the definition of $r_{A}$ imply the existence of a set $B \subset A \cap(\delta, \infty)$ of positive Lebesgue measure so that $\left(p_{i}\right)_{i=1}^{\infty}$ converges uniformly on $B$, hence it is uniformly Cauchy on $B$. Now Theorem 5.1 yields that $\left(p_{i}\right)_{i=1}^{\infty}$ is uniformly Cauchy on $[0, \delta]$, which proves the theorem.

Theorem 6.4. Suppose $\sum_{i=1}^{\infty} 1 / \lambda_{i}<\infty, A \subset[0, \infty)$ is a set of positive Lebesgue measure, $w$ is a non-negative-valued, integrable weight function on $A$ with $\int_{A} w>0$, and $q \in(0, \infty)$. Then $M(\Lambda)$ is not dense in $L_{w}^{q}(A)$.

Moreover, if the gap condition (6.1) holds, then every function $f \in L_{w}^{q}(A)$ belonging to the $L_{w}^{q}(A)$ closure of $M(\Lambda)$ can be represented as

$$
f(x)=\sum_{i=0}^{\infty} a_{i} x^{\lambda_{i}}, \quad x \in A \cap\left[0, r_{w}\right),
$$

where

$$
r_{w}:=\sup \left\{x \in[0, \infty): \int_{A \cap(x, \infty)} w(x) d x>0\right\} .
$$


If the gap condition (6.1) does not hold, then every function $f \in L_{w}^{q}(A)$ belonging to the $L_{w}^{q}(A)$ closure of $M(\Lambda)$ can still be represented as an analytic function on

$$
\left\{z \in \mathbb{C} \backslash(-\infty, 0]:|z|<r_{w}\right\}
$$

restricted to $A$.

Proof. Suppose $f \in L_{w}^{q}(A)$ and suppose there is a sequence $\left(p_{i}\right)_{i=1}^{\infty} \subset M(\Lambda)$ so that

$$
\lim _{i \rightarrow \infty}\left\|f-p_{i}\right\|_{L_{w}^{q}(A)}=0
$$

Minkowski's inequality (if $q \in(0,1)$, then a multiplicative factor $2^{1 / q-1}$ is needed) yields that $\left(p_{i}\right)_{i=1}^{\infty}$ is a Cauchy sequence in $L_{w}^{q}(A)$. The assumptions on $w$ imply that for every $\delta \in\left(0, r_{w}\right)$ there exists an $\alpha>0$ so that

$$
B:=\{x \in A \cap(\delta, \infty): w(x)>\alpha\}
$$

is of positive Lebesgue measure. Note that

$$
\|p\|_{L^{q}(B)} \leq \alpha^{-1}\|p\|_{L_{w}^{q}(B)} \leq \alpha^{-1}\|p\|_{L_{w}^{q}(A)}
$$

for every $p \in L_{w}^{q}(A)$. Therefore, $\left(p_{i}\right)_{i=1}^{\infty}$ is a Cauchy sequence in $L^{q}(B)$. So, by Theorem 5.1, $\left(p_{i}\right)_{i=1}^{\infty}$ is uniformly Cauchy on $[0, \delta]$. If the gap condition (6.1) holds, then the theorem now follows from results of Clarkson and Erdoss [12] (see the end of the proof of Theorem 6.1). If the gap condition (6.1) does not hold, then a result of Schwartz [24] yields the theorem (see also the end of the proof of Theorem $6.1)$.

Theorem 6.5. Suppose $A \subset[0,1]$ is a set of positive Lebesgue measure, $w$ is a non-negative-valued integrable weight function on $A$ with $\int_{A} w>0$, and $q \in(0, \infty)$. Then $M(\Lambda)$ is dense in $L_{w}^{q}(A)$ if and only if $\sum_{i=1}^{\infty} 1 / \lambda_{i}=\infty$.

Proof. Suppose $\sum_{i=1}^{\infty} 1 / \lambda_{i}=\infty$. Let $f \in L_{w}^{q}(A)$. It is standard measure theory to show that for every $\varepsilon>0$, there exists a $g \in C[0,1]$ so that

$$
\|f-g\|_{L_{w}^{q}(A)}<\frac{\varepsilon}{2} .
$$

Now Müntz's Theorem implies that there exists a $p \in M(\Lambda)$ so that

$$
\|g-p\|_{L_{w}^{q}(A)} \leq\|g-p\|_{A}\left(\int_{A} w\right)^{1 / q}<\frac{\varepsilon}{2} .
$$

Therefore $M(\Lambda)$ is dense in $L_{w}^{q}(A)$.

Suppose now that $\sum_{i=1}^{\infty} 1 / \lambda_{i}<\infty$. Then Theorem 6.4 yields that $M(\Lambda)$ is not dense in $L_{w}^{q}(A)$.

\section{Products of Müntz spaces}

For

$$
\Lambda_{j}:=\left(\lambda_{i, j}\right)_{i=0}^{\infty}, \quad 0=\lambda_{0, j}<\lambda_{1, j}<\lambda_{2, j}<\cdots, \quad j=1,2, \ldots,
$$

we define the sets

$$
M\left(\Lambda_{1}, \Lambda_{2}, \ldots, \Lambda_{k}\right):=\left\{p=\prod_{j=1}^{k} p_{j}: p_{j} \in M\left(\Lambda_{j}\right)\right\} .
$$

First we prove the following Remez-type inequality for $M\left(\Lambda_{1}, \Lambda_{2}, \ldots, \Lambda_{k}\right)$. 
Theorem 7.1. Suppose (7.1) holds and

$$
\sum_{i=1}^{\infty} \frac{1}{\lambda_{i, j}}<\infty, \quad j=1,2, \ldots, k .
$$

Let $s>0$. Then there exists a constant $c$ depending only on $\Lambda_{1}, \Lambda_{2}, \ldots, \Lambda_{k}, s$, and $k$ (and not on $\varrho$ or $A$ ) so that

$$
\|p\|_{[0, \varrho]} \leq c\|p\|_{A}
$$

for every $p \in M\left(\Lambda_{1}, \Lambda_{2}, \ldots, \Lambda_{k}\right)$ and for every set $A \subset[\varrho, 1]$ of Lebesgue measure at least $s$.

Proof. Theorem 5.1 implies that there exist constants $\alpha_{j}>0$ depending only on $\Lambda_{1}, \Lambda_{2}, \ldots, \Lambda_{k}, s$, and $k$ so that

$$
m\left(\left\{x \in[y, 1]:|p(x)|>\alpha_{j}^{-1}|p(y)|\right\}\right) \geq 1-y-\frac{s}{2 k}
$$

for every $p \in M\left(\Lambda_{j}\right)$ and $y \in[0,1-s]$. Indeed, $\alpha_{j}:=c+1$ is a suitable choice, where $c$ is the constant in Theorem 5.1 depending only on $\Lambda:=\Lambda_{j}$ and $\tilde{s}:=s /(2 k)$. Otherwise, if

$$
m\left(\left\{x \in[y, 1]:|p(x)|>(c+1)^{-1}|p(y)|\right\}\right)<1-y-\frac{s}{2 k}
$$

for some $p \in M\left(\Lambda_{j}\right)$, then, on one hand, $|p(y)|>0$ would hold, while on the other hand, Theorem 5.1 with $\left.A:=\left\{x \in[y, 1]:|p(x)| \leq(c+1)^{-1}|p(y)|\right\}\right)$ and $m(A) \geq s /(2 k)$ would imply that $|p(y)| \leq c(c+1)^{-1}|p(y)|$. This is a contradiction, so the existence of the constants $\alpha_{j}$ with the desired properties are justified.

Now let

$$
p \in M\left(\Lambda_{1}, \Lambda_{2}, \ldots, \Lambda_{k}\right),
$$

that is,

$$
p=\prod_{j=1}^{k} p_{j}, \quad p_{j} \in M\left(\Lambda_{j}\right) .
$$

Then, for every $y \in[0,1-s]$,

$$
\begin{aligned}
m(\{x \in[y, 1]:|p(x)| & \left.\left.>\left(\alpha_{1} \alpha_{2} \cdots \alpha_{k}\right)^{-1}|p(y)|\right\}\right) \\
\geq & m\left(\bigcap_{j=1}^{k}\left\{x \in[y, 1]:\left|p_{j}(x)\right|>\alpha_{j}^{-1}\left|p_{j}(y)\right|\right\}\right) \\
\geq & 1-y-k \frac{s}{2 k}=1-y-\frac{s}{2} .
\end{aligned}
$$

Hence $y \in[0, \inf A]$ and $m(A) \geq s$ imply

$$
m\left(\left\{x \in A:|p(x)|>\left(\alpha_{1} \alpha_{2} \cdots \alpha_{k}\right)^{-1}|p(y)|\right\}\right) \geq \frac{s}{2}>0,
$$

and the theorem follows with $c:=\alpha_{1} \alpha_{2} \cdots \alpha_{k}$.

Theorem 7.1 immediately solves Newman's problem [20].

Corollary 7.2. Suppose (7.1) and (7.2) hold and $A \subset[0,1]$ is a set of positive Lebesgue measure. Then $M\left(\Lambda_{1}, \Lambda_{2}, \ldots, \Lambda_{k}\right)$ is not dense in $C(A)$. Moreover, if $w$ is a non-negative-valued integrable weight function on $A$ with $\int_{A} w>0$ and $q \in(0, \infty)$, then $M\left(\Lambda_{1}, \Lambda_{2}, \ldots, \Lambda_{k}\right)$ is not dense in $L_{w}^{q}(A)$. 
Our next theorem establishes a Bernstein-type inequality for $M\left(\Lambda_{1}, \Lambda_{2}, \ldots, \Lambda_{k}\right)$.

Theorem 7.3. Suppose (7.1) and (7.2) hold and

$$
\lambda_{1, j} \geq 1, \quad j=1,2, \ldots, k .
$$

Let $s>0$. Then there exists a constant $c$ depending only on $\Lambda_{1}, \Lambda_{2}, \ldots, \Lambda_{k}$, $s$, and $k$ (and not on $\varrho$ or $A$ ) so that

$$
\left\|p^{\prime}\right\|_{[0, \varrho]} \leq c\|p\|_{A}
$$

for every $p \in M\left(\Lambda_{1}, \Lambda_{2}, \ldots, \Lambda_{k}\right)$ and for every set $A \subset[\varrho, 1]$ of Lebesgue measure at least $s$.

Proof. Note that a combination of Theorems 5.1 and 3.2 implies that there exist constants $\beta_{j}>0$ depending only on $\Lambda_{1}, \Lambda_{2}, \ldots, \Lambda_{k}, s$, and $k$ so that

$$
m\left(\left\{x \in[y, 1]:|p(x)|>\beta_{j}^{-1}\left|p^{\prime}(y)\right|\right\}\right) \geq 1-y-\frac{s}{2 k}
$$

for every $p \in M\left(\Lambda_{j}\right)$ and $y \in[0,1-s]$. Indeed, $\beta_{j}:=\left(c_{1}+1\right) c_{2}$ is a suitable choice, where $c_{1}$ is the constant in Theorem 5.1 depending only on $\Lambda:=\Lambda_{j}$ and $\tilde{s}:=s /(4 k)$, while $c_{2}$ is a constant depending only on $\Lambda:=\Lambda_{j}$ and $s /(4 k)$ so that

$$
\left|p^{\prime}(y)\right| \leq c_{2}\|p\|_{[0, y+s /(4 k)]},
$$

the existence of which follows from Theorem 3.2 by the scaling $x \mapsto(y+s /(4 k)) x$. To see that $\beta_{j}$ above is a suitable choice, suppose that

$$
m\left(\left\{x \in[y, 1]:|p(x)|>\left(c_{2}\left(c_{1}+1\right)\right)^{-1}\left|p^{\prime}(y)\right|\right\}\right)<1-y-\frac{s}{2 k}
$$

for some $p \in M\left(\Lambda_{j}\right)$. Then $\left|p^{\prime}(y)\right|>0$. Let

$$
A:=\left\{x \in[y+s /(4 k), 1]:|p(x)| \leq\left(\left(c_{1}+1\right) c_{2}\right)^{-1}\left|p^{\prime}(y)\right|\right\} .
$$

Then $m(A) \geq s /(2 k)-s /(4 k)=s /(4 k)$, and by Theorem 5.1 ,

$$
\|p\|_{[0, y+s /(4 k)]} \leq c_{1}\left(c_{1}+1\right)^{-1} c_{2}^{-1}\left|p^{\prime}(y)\right|<c_{2}^{-1}\left|p^{\prime}(y)\right| .
$$

This contradicts the choice of $c_{2}$. Therefore the existence of the constants $\beta_{j}$ with the desired properties are justified.

Let $\alpha_{j}>0, j=1,2, \ldots, k$, be chosen as in the proof of Theorem 7.1. Now let

$$
p \in M\left(\Lambda_{1}, \Lambda_{2}, \ldots, \Lambda_{k}\right),
$$

that is,

$$
p=\prod_{j=1}^{k} p_{j}, \quad p_{j} \in M\left(\Lambda_{j}\right) .
$$

Then, as in the proof of Theorem 7.1 , for every $y \in[0,1-s]$,

$$
\begin{gathered}
m\left(\bigcap_{j=1}^{k}\left\{x \in[y, 1]:|p(x)|>\gamma_{j}^{-1}\left|\left(p_{1} \cdots p_{j-1} p_{j}^{\prime} p_{j+1} \cdots p_{k}\right)(y)\right|\right\}\right) \\
\geq 1-y-k \frac{s}{2 k}=1-y-\frac{s}{2}
\end{gathered}
$$


where

$$
\gamma_{j}:=\alpha_{1} \cdots \alpha_{j-1} \beta_{j} \alpha_{j+1} \cdots \alpha_{k}
$$

So

$$
m\left\{x \in[y, 1]:|p(x)|>c^{-1}\left|p^{\prime}(y)\right|\right\} \geq 1-y-\frac{s}{2},
$$

where $c:=\sum_{j=1}^{k} \gamma_{j}$. Hence $y \in[0, \inf A]$ and $m(A) \geq s$ imply

$$
m\left(\left\{x \in A:|p(x)|>c^{-1}\left|p^{\prime}(y)\right|\right\}\right) \geq \frac{s}{2}>0,
$$

and the theorem follows with $c=\sum_{j=1}^{k} \gamma_{j}$.

The following Nikolskii-type inequality is also valid for $M\left(\Lambda_{1}, \Lambda_{2}, \ldots, \Lambda_{k}\right)$.

Theorem 7.4. Suppose (7.1) and (7.2) hold. Let $s>0$ and $q \in(0, \infty)$. Then there exists a constant $c$ depending only on $\Lambda_{1}, \Lambda_{2}, \ldots, \Lambda_{k}, s, k, q$, and $w$ (and not on $\varrho$ or $A$ ) so that

$$
\|p\|_{[0, \varrho]}^{q} \leq c \int_{A}|p(x)|^{q} w(x) d x
$$

for every $p \in M\left(\Lambda_{1}, \Lambda_{2}, \ldots, \Lambda_{k}\right)$, for every set $A \subset[\varrho, 1]$ of Lebesgue measure at least $s$, and for every function $w$ measurable and positive a.e. on $[0,1]$.

Proof. This a straightforward consequence of Theorem 7.1

\section{ACKNowledgement}

The authors thank Yuri Brudnyi for sending them a translation of Remez's paper $[22]$.

\section{REFERENCES}

1. J. M. Anderson, Müntz-Szász type approximation and the angular growth of lacunary integral functions, Trans. Amer. Math. Soc. 169 (1972), 237-248. MR 46:9360

2. J. Bak and D. J. Newman, Rational combinations of $x^{\lambda_{k}}, \lambda_{k} \geq 0$ are always dense in $C[0,1]$, J. Approx. Theory 23 (1978), 155-157. MR 58:6840

3. S. N. Bernstein, Collected Works: Vol 1. Constructive Theory of Functions (1905-1930), English Translation, Atomic Energy Commission, Springfield, Va, 1958. MR 14:2c

4. R. P. Boas, Entire Functions, Academic Press, New York, 1954. MR 16:914f

5. P. B. Borwein, Zeros of Chebyshev polynomials in Markov Systems, J. Approx. Theory 63 (1990), 56-64. MR 92a:41002

6. P. B. Borwein, Variations on Müntz's theme, Can. Math. Bull. 34 (1991), 305-310. MR 92i: 41010

7. P. B. Borwein and T. Erdélyi, Notes on lacunary Müntz polynomials, Israel J. Math. 76 (1991), 183-192. MR 93h:41014

8. P. B. Borwein and T. Erdélyi, Lacunary Müntz systems, Proc. Edinburgh Math. Soc. 36 (1993), 361-374. MR 94i:41023

9. P. B. Borwein and T. Erdélyi, Polynomials and Polynomial Inequalities, Springer-Verlag, New York, 1995. CMP 96:06

10. P. B. Borwein, T. Erdélyi, and J. Zhang, Müntz systems and orthogonal Müntz polynomials, Trans. Amer. Math. Soc. 342 (1994), 523-542. MR 94f:42026

11. E. W. Cheney, Introduction to Approximation Theory, McGraw-Hill, New York, 1966. MR 36:5568 
12. J. A. Clarkson and P. Erdős, Approximation by polynomials, Duke Math. J. 10 (1943), 5-11. MR 4:196e

13. T. Erdélyi, Remez-type inequalities on the size of generalized polynomials, J. London Math. Soc. 45 (1992), 255-264. MR 93e:41022

14. T. Erdélyi, Remez-type inequalities and their applications, J. Comp. and Applied Math. 47 (1993), 167-210. MR 94m:26003

15. G. Freud, Orthogonal Polynomials, Pergamon Press, Oxford, 1971.

16. S. Karlin and W. J. Studden, Tchebycheff Systems with Applications in Analysis and Statistics, Wiley, New York, 1966. MR 34:4757

17. W. A. J. Luxemburg and J. Korevaar, Entire functions and Müntz-Szász type approximation, Trans. Amer. Math. Soc. 157 (1971), 23-37. MR 43:7643

18. C. Müntz, Über den Approximationsatz von Weierstrass, H. A. Schwartz Festschrift, Berlin, 1914.

19. D. J. Newman, Derivative bounds for Müntz polynomials, J. Approx. Theory 18 (1976), 360362. MR 55:3609

20. D. J. Newman, Approximation with rational functions, vol. 41, Regional Conference Series in Mathematics, Providence, Rhode Island, 1979. MR 84k:41019

21. G. Nürnberger, Approximation by Spline Functions, Springer-Verlag, Berlin, 1989. MR 90j: 41025

22. E. J. Remez, Sur une propriété des polynômes de Tchebyscheff, Comm. Inst. Sci. Kharkow 13 (1936), 93-95.

23. T. J. Rivlin, Chebyshev Polynomials, 2nd ed., Wiley, New York, 1990. MR 92a:41016

24. L. Schwartz, Etude des Sommes d'Exponentielles, Hermann, Paris, 1959. MR 21:5116

25. P. W. Smith, An improvement theorem for Descartes systems, Proc. Amer. Math. Soc. 70 (1978), 26-30. MR 57:6985

26. G. Somorjai, A Müntz-type problem for rational approximation, Acta. Math. Hung. 27 (1976), 197-199. MR 55:3622

27. O. Szász, Über die Approximation steliger Funktionen durch lineare Aggregate von Potenzen, vol. 77,1916 , pp. $482-496$.

28. G. Szegö, On the density of quotients of lacunary polynomials, Acta Math. Hung. 30 (1922), 149-154.

29. A. K. Taslakyan, Some properties of Legendre quasi-polynomials with respect to a Müntz system, Mathematics 2 (1984), 179-189; Erevan University, Erevan. (Russian, Armenian Summary) MR 88e:33008

30. M. von Golitschek, A short proof of Müntz Theorem, J. Approx. Theory 39 (1983), 394-395. MR 85b:41005

ABStRACT. The principal result of this paper is a Remez-type inequality for Müntz polynomials:

$$
p(x):=\sum_{i=0}^{n} a_{i} x^{\lambda_{i}},
$$

or equivalently for Dirichlet sums:

$$
P(t):=\sum_{i=0}^{n} a_{i} e^{-\lambda_{i} t},
$$

where $0=\lambda_{0}<\lambda_{1}<\lambda_{2}<\cdots$. The most useful form of this inequality states that for every sequence $\left(\lambda_{i}\right)_{i=0}^{\infty}$ satisfying $\sum_{i=1}^{\infty} 1 / \lambda_{i}<\infty$, there is a constant $c$ depending only on $\Lambda:=\left(\lambda_{i}\right)_{i=0}^{\infty}$ and $s$ (and not on $n, \varrho$, or $A$ ) so that

$$
\|p\|_{[0, \varrho]} \leq c\|p\|_{A}
$$

for every Müntz polynomial $p$, as above, associated with $\left(\lambda_{i}\right)_{i=0}^{\infty}$, and for every set $A \subset[\varrho, 1]$ of Lebesgue measure at least $s>0$. Here $\|\cdot\|_{A}$ denotes the supremum norm on $A$. This Remez-type inequality allows us to resolve two reasonably long-standing conjectures.

The first conjecture it lets us resolve is due to D. J. Newman and dates from 1978. It asserts that if $\sum_{i=1}^{\infty} 1 / \lambda_{i}<\infty$, then the set of products $\left\{p_{1} p_{2}\right.$ : $\left.p_{1}, p_{2} \in \operatorname{span}\left\{x^{\lambda_{0}}, x^{\lambda_{1}}, \ldots\right\}\right\}$ is not dense in $C[0,1]$. 
The second is a complete extension of Müntz's classical theorem on the denseness of Müntz spaces in $C[0,1]$ to denseness in $C(A)$, where $A \subset[0, \infty)$ is an arbitrary compact set with positive Lebesgue measure. That is, for an arbitrary compact set $A \subset[0, \infty)$ with positive Lebesgue measure, $\operatorname{span}\left\{x^{\lambda_{0}}, x^{\lambda_{1}}, \ldots\right\}$ is dense in $C(A)$ if and only if $\sum_{i=1}^{\infty} 1 / \lambda_{i}=\infty$.

Several other interesting consequences are also presented.

Department of Mathematics, Simon Fraser University, Burnaby, British Columbia, CANADA V5A 1 S6

E-mail address: pborwein@cecm.sfu.ca

Department of Mathematics, Texas A\&M University, College Station, Texas 778433368

E-mail address: terdelyi@math.tamu.edu 